\section{A MATÉRIA MOLE E A LUZ SINGROTRON}

\author{
Watson Loh \\ Nádya Pesce da Silveira
}

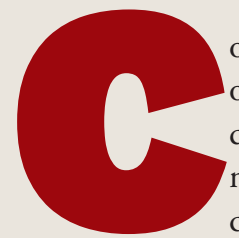

om o advento da nanociência e da nanotecnologia o mundo científico passou a utilizar mais o conceito de matéria mole - soft matter na língua inglesa. Essa nomenclatura é utilizada há décadas pelos cientistas que realizam pesquisas em ciência básica envolvendo materiais moleculares das mais diversas origens. Trata-se de materiais que se auto-organizam, como resultado de interações físicas não-covalentes, originando sistemas químicos de grande interesse biomédico e tecnológico, na forma de materiais funcionais (1). De acordo com De Gennes (2), prêmio Nobel de física em 1991, a matéria mole se caracteriza pelo fato de, em sofrendo pequenas modificaçôes químicas, apresentar grandes mudanças em suas características mecânicas. A matéria mole também se caracteriza por apresentar flutuaçôes térmicas em temperaturas próximas da temperatura ambiente. Originalmente, os principais materiais envolvidos no conceito de matéria mole são polímeros, cristais líquidos e surfactantes, todos classificados como sistemas coloidais (2). Costumamos relacionar com a matéria mole os sistemas moleculares ou supramoleculares que são capazes de responder a estímulos externos de diferentes naturezas, tais como pressão, temperatura e $\mathrm{pH}$, dentre outros.

Com a introdução de grandes equipamentos no estudo de materiais, especialmente a utilização das técnicas de espalhamento (de luz, raios X e nêutrons), a compreensão das escalas mesoscópicas (dimensões que se situam entre o regime atômico-molecular e o limite macroscópico) e suas dinâmicas na matéria mole ganhou grandes aliados. Isso deve-se ao fato de que as técnicas de espalhamento fornecem informaçōes estatísticas acerca das diferentes dimensões dessas estruturas supramoleculares e permitem o estudo das propriedades estáticas e dinâmicas de materiais baseados em matéria mole, que são de interesse científico e tecnológico (1). A manutenção e ampliação das fontes de radiação síncrotron, como aquela disponibilizada no Laboratório Nacional de Luz Síncrotron (LNLS) em Campinas, vem permitindo especialmente o avanço das técnicas de espalhamento de raios X utilizadas no estudo da matéria mole, com destaque para o espalhamento de raios $\mathrm{X}$ em ângulos baixos (SAXS). Uma das principais características do SAXS realizado com luz síncrotron, quando comparado a outros métodos espectroscópicos utilizados no estudo de materiais, é que a técnica possibilita a resolução espacial da matéria mole a nível nanométrico e sub-micrométrico, bem como a resolução de processos temporais (dinâmicos) que ocorrem ao nível do milissegundo/segundo (3). Por outro lado, similarmente ao que ocorre com a técnica de espalhamento de luz dinâmico (DLS), utilizada para elucidar processos dinâmicos detectáveis na região da luz visível, o experimento de espalhamento dinâmico de raios $\mathrm{X}$ coerentes, denominado espectroscopia de correlação de fótons de raios X (XPCS), permite o estudo de processos dinâmicos na região de tamanhos estudada por SAXS.

O projeto Sirius (4), em implementação no Centro Nacional de Pesquisa em Energia e Materiais (CNPEM), onde está localizado o LNLS, em Campinas, permitirá a consolidação de uma nova fonte de luz síncrotron brasileira. O Sirius será uma das principais fontes de luz síncrotron de quarta geração do mundo. Uma das linhas de luz a ser desenvolvida no Sirius é a de espectroscopia de correlação de fótons de raios X (XPCS), que permitirá um grande avanço nos estudos dedicados à físico-química da matéria mole. A seguir, discorreremos brevemente sobre o princípio das técnicas espectroscópicas de espalhamento, bem como sobre alguns sistemas de interesse tecnológico que são majoritariamente investigados utilizando a luz síncrotron, através da técnica de SAXS. Alguns desses sistemas poderão ter suas principais propriedades dinâmicas elucidadas através da técnica de XPCS, em implementação no CNPEM.

\section{A MATÉRIA MOLE E A ESPECTROSCOPIA DE ESPALHAMENTO DE RAIOS X}

A técnica de espalhamento de raios $\mathrm{X}$ aplicada a sistemas meso-estruturados (que contêm um certo grau de organização, possuindo dimensōes nanométricas a micrométricas) utiliza como fundamento o princípio da interferência das ondas eletromagnéticas espalhadas por diferentes pontos em um mesmo material (5). Pode-se dizer que o espalhamento das ondas pelos materiais origina-se das flutuações espaciais da densidade de elétrons nesses materiais. Em experimentos utilizando espalhamento de raios $\mathrm{X}$, a radiação espalhada é observada a uma distância relativamente grande da amostra (que é irradiada com raios $\mathrm{X}$ ) e em diferentes ângulos de observação (em relação ao feixe de radiação que incide na amostra) que variam, tipicamente, de 0,1 a 10 graus. $\mathrm{O}$ experimento de espalhamento de raios $\mathrm{X}$ em ângulos baixos (SAXS) utiliza, geralmente, um detector bidimensional, através do qual pode-se relacionar o quadrado da amplitude da onda espalhada (que chega ao detector) com o número de fótons produzidos. No experimento de SAXS, as ondas interferentes geralmente são originadas de pontos intra-partícula (centros espalhadores de um exemplar médio da amostra), pois o comprimento da radiação utilizada (raios X) possui dimensão próxima às estruturas componente

Dessa forma, a técnica de SAXS é muito utilizada para elucidar a dimensão de estruturas componentes de um mesmo sistema espalhador (amostra), sendo menos aplicada ao dimensionamento de partículas nanométricas. Entretanto, uma análise por modelagem do perfil das curvas de radiação espalhada obtidas experimentalmente permite a determinação da dimensão aproximada de partículas, das distâncias entre as partículas, e até mesmo a elucidação do tipo de geometria de uma determinada partícula (esférica, tubular etc.). Para caracterizar todos esses aspectos de um mesmo sistema (amostra) utilizando a técnica de SAXS, a análise dos dados experi- 
mentais obtidos necessita ser feita com aplicação de modelos teóricos previamente estabelecidos.

Nos experimentos de SAXS com radiação síncrotron são utilizados raios X com comprimentos de onda $(\lambda)$ entre 0,01 e 0,4 nm e as energias dos feixes de raios $\mathrm{X}$ incidentes na amostra são da ordem de 10 $\mathrm{keV}(5)$. A diferença de fase entre as ondas espalhadas pela amostraéde aproximadamente $0,1 \mathrm{~nm}^{-1}$. Energias ainda mais elevadas podem ser utilizadas em experimentos específicos, nos quais a largura dos feixes de raios $\mathrm{X}$ incidentes também pode ser modificada. Uma vez que as ondas eletromagnéticas incidentes e espalhadas na amostra podem ser representadas como vetores, é possível definir a diferença entre as mesmas como sendo o vetor de onda (q), cuja dimensão depende do comprimento de onda $(\lambda)$ da radiação utilizada e do ângulo $(\theta)$ de observação da radiação espalhada: $q=4 \pi / \lambda(\operatorname{sen} \theta / 2)$. Nos experimentos de SAXS, a região de $q$ acessada varia de 0,006 a $6 \mathrm{~nm}^{-1}$.

Tipicamente, a técnica de SAXS permite a extração de informaçôes sobre a média estatística de um volume de aproximadamente 1 $\mathrm{mm}^{3}$ (6). As dimensōes (superestrutura de um dado sistema que chamamos de amostra) determinadas por SAXS ficam entre $1 \mathrm{~nm}$ e aproximadamente $1 \mu \mathrm{m}$, sendo dessa forma ideal para o estudo de sistemas coloidais. Assim, a técnica de SAXS convencional permite relacionar a intensidade média da radiação espalhada estaticamente pelos componentes de uma amostra $\left(\mathrm{I}_{q}\right)$ com a sua mesoestrutura (composta de dimensōes que se situam entre o regime atômico-molecular e o limite macroscópico). Ainda, utilizando radiação (raios X) altamente coerente, e se a intensidade da radiação espalhada pela amostra é medida em uma escala de tempo comparável a uma dinâmica característica do sistema em estudo, é possível também obter informações sobre as dinâmicas intrínsecas do sistema (amostra) em determinadas condições experimentais. Nesse caso estaremos realizando um experimento de espectroscopia de correlação de fótons de raios X (XPCS).

Convencionalmente, XPCS baseia-se na forma como um material relativamente desorganizado origina um padrão de espalhamento. Esses padróes de espalhamento possuem um tamanho determinado e originam-se da interferência construtiva do espalhamento coerente a partir de centros espalhadores aleatoriamente distribuídos na amostra (7). Em função do movimento browniano (aleatório) dos centros espalhadores, os padrões de espalhamento mudam com o tempo, permitindo a detecção da dinâmica intrínseca do sistema em análise (amostra). Na prática, um arranjo aleatório de unidades espalhadoras componentes da amostra é atingida por uma radiação coerente (no caso, raios X) e a intensidade espalhada pode ser analisada na forma de uma imagem espacial, a qual está relacionada com a configuração momentânea das unidades espalhadoras da matéria mole (amostra) em questão.

O movimento (dinâmica) dessas unidades causará uma modificação da imagem. Uma correlação entre a imagem final e a inicial conterá informaçōes sobre as dinâmicas características daquele sistema em análise (amostra). A técnica de XPCS é idealmente aplicada para estudar a relação entre cinética e estrutura na matéria mole. Uma das

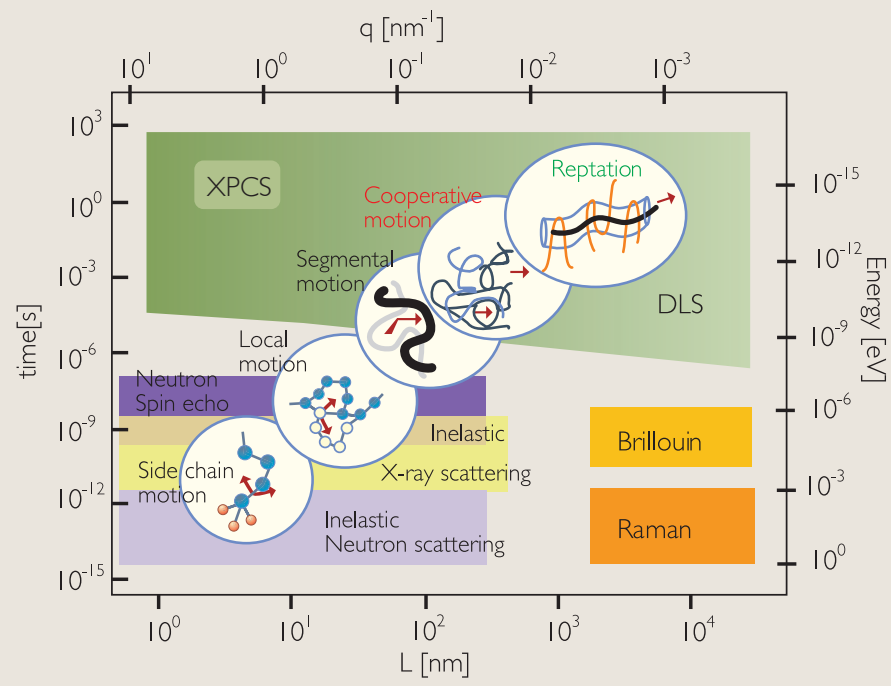

Figura 1. Representação esquemática da relação entre dinâmica (eixo y) e dimensões (eixo $x$ ) características definindo regiões que podem ser investigadas em experimentos de SAXS, DLS e XPCS. Reproduzida de (12) com permissão de Takahara

grandes vantagens da técnica é poder medir diretamente as flutuações termodinâmicas características de um determinado sistema de interesse (amostra). São essas flutuaçōes termodinâmicas que permitem a evolução de um determinado sistema em função do tempo quando a matéria sofre estímulos externos, tais como pressão, temperatura e $\mathrm{pH}$, dentre outros. Tipicamente, os tempos de relaxação (dinâmica) determinados por XPCS situam-se entre $10^{-10}$ e $10^{2} s$ (Figura 1), correspondendo a dimensões que variam do nanômetro ao milímetro.

\section{EXEMPLOS DE SISTEMAS COLOIDAIS INVESTIGADOS POR SAXS E XPCS} Agregados de tensoativos Tensoativos ou surfactantes são moléculas que apresentam caráter anfifílico em relação ao solvente e, por este motivo, tendem a se agregar de modo a diminuir a energia do sistema pela diminuição de contatos desfavoráveis com o solvente. O tipo mais simples de estrutura assim formada é a micela, que representa um arranjo próximo do esférico decorrente da agregação de surfactantes, com tamanho de cerca de poucos nanômetros. Soluçôes mais concentradas de surfactantes tendem a apresentar outros arranjos geométricos, tais como fases lamelares, cúbicas e hexagonais. Genericamente, essas são chamadas de mesofases ou fases líquido-cristalinas por apresentarem ordem, mas apenas de curto alcance, se considerado o sistema como um todo.

A formação de agregados mais complexos é um processo cuja dinâmica foi apenas recentemente investigada em um estudo que aplicou a técnica de SAXS resolvida no tempo para acompanhar a transição de forma de uma micela esférica para uma micela cilíndrica alongada. $\mathrm{O}$ trabalho revelou que o processo deve ocorrer a partir de sucessivos processos de fusão dos agregados globulares, formando agregados cada vez mais alongados, em uma escala de tempo de até dezenas de nanosegundos (8). 
Técnicas como SAXS são apropriadas para caracterização estrutural das mesofases de surfactantes, sendo normalmente utilizadas para elucidação, por exemplo, de diagramas de fase de surfactantes. Um exemplo dessa aplicação pode ser verificado em estudo envolvendo misturas de surfactantes e copolímeros-em-bloco com cargas opostas (9). Nesse sistema, formado pelo surfactante dodeciltrimetilamônio e copolímero poli(acrilato-b-acrilamida), formam-se nanopartículas em que o interior tem arranjo líquido-cristalino. A adição de quantidades crescentes de n-octanol leva a um rearranjo dessa estrutura interna, formando todas as mesofases mais comuns, inclusive as fases reversas (em que a água está contida no interior dos agregados). Nesse caso, a técnica de SAXS é a única que permite identificar inequivocamente as estruturas formadas, além de fornecer as dimensões desses arranjos. Uma conclusão interessante desse estudo é que essas dimensões não são afetadas pelo confinamento no interior da nanopartícula.

Lipossomas são outras estruturas coloidais formadas pela auto-organização de moléculas lipídicas (do tipo fosfolipídeos) em solução. São estruturas supramoleculares utilizadas na pesquisa científica básica como modelos de membranas biológicas ou na forma de reatores em nano-escala, por exemplo. Na última década, o desenvolvimento de lipossomas compósitos contendo polieletrólitos associados a lipossomas apresentou implicações científicas e tecnológicas $(10,11)$. O emprego da quitosana como polieletrólito deu origem aos chamados quitossomas, e aprimorou a estabilidade dos lipossomas tradicionais compostos apenas de lipídeos, bem como suas características físico-químicas e superficiais (10). Na área de alimentos, esses sistemas já são empregados como estabilizantes; e na área farmacêutica, como carreadores de substâncias bioativas e na produção de vacinas (11). Esses sistemas são preparados a partir da obtenção de um núcleo fosfolipídico, o qual é revestido pela quitosana. Mesmo em pequenas quantidades, a quitosana mostrou-se capaz de promover a estabilização da parede lipídica dos lipossomas através de interações eletrostáticas entre os grupamentos fosfato dos fosfolipídeos e o grupo amino da quitosana.

Como as técnicas de espalhamento - como SAXS - são bastante utilizadas para a determinação da estrutura interna de sistemas nanométricos auto-organizados do tipo coloidal, a avaliação do impacto de diferentes quantidades de quitosana sobre o tamanho dos quitossomas, o número e a rigidez das bicamadas lipídicas nos quitossomas foi realizada utilizando-se a técnica de SAXS em combinação com DLS (dynamic lightscattering). Por meio da análise dos resultados aplicando ajustes teóricos às curvas (por exemplo, analisando o pico principal no espectro de SAXS como uma função matemática lorentziana) e modelos matemáticos (por exemplo, utilizando o modelo teórico de Caillé) foi possível determinar que a espessura da bicamada lipídica nos quitossomas não variou grandemente conforme aumentou-se a quantidade de quitosana nas preparaçôes, permanecendo em torno de $6,5 \pm 0,1 \mathrm{~nm}$. Entretanto, o número de bicamadas lipídicas em cada quitossoma aumentou de aproximadamente 1 (unilamelar) para 12 (multilamelar), quando se variou a quantidade de quitosana utilizada nas preparações. Já o parâmetro de Caillé - que corresponde a uma medida das flutuações de deformação na bicamada lipídica originada nas ondulações presentes naturalmente nas membranas em função da temperatura-variou de 0,32 a 0,27 com o aumento da quantidade de quitosana presente na formulação. Esses resultados apontam para uma maior rigidez da membrada lipídica dos quitossomas pelo acréscimo do polieletrólito. A técnica de XPCS poderá ser útil na elucidação da ordem de grandeza das flutuações presentes em membranas lipídicas, contribuindo para a determinação da estabilidade mecânica de tais membranas, quando da sua aplicação.

Polímeros Superfícies e interfaces presentes na matéria mole podem ser de grande importância na aplicação de materiais poliméricos. Superfícies contendo organizaçōes poliméricas do tipo escova, por exemplo, são reconhecidas como de grande importância tecnológica e têm sido objeto de estudos científicos bastante avançados, com o intuito de reconhecer todas as suas propriedades intrínsecas (12). O conhecimento sobre a dinâmica de tais materiais é muito importante para sua aplicabilidade. Devido à complexidade desses sistemas em nível molecular, sua natureza compreende um grande intervalo de dimensões e tempos característicos (dinâmicas). Dentro dessa perspectiva, os métodos de espalhamento de radiação são ferramentas muito importantes na sua caracterização. Um artigo de revisão publicado recentemente (12) ilustra perfeitamente todos os aspectos mais importantes do estudo desse tipo de matéria mole, quando se trata de elucidar suas características dinâmicas utilizando a técnica de espectroscopia de correlação de fótons de raios X(XPCS). Através dessa técnica, considerada essencial na compreensão do comportamento das escovas poliméricas, os movimentos típicos de segmentos das cadeias e o movimento cooperativo entre os mesmos podem ser determinados. O trabalho ilustra claramente a correlação entre as dimensões das cadeias compostas por organizações poliméricas do tipo escova e seus movimentos característicos, inclusive exemplificando o que outras técnicas, como o DLS, podem oferecer (12). Os autores consideram que muitas das propriedades dos materiais do tipo escovas poliméricas, tais como capacidade de adesão em superfícies, molhabilidade etc., estão intrinsicamente relacionadas com as propriedades superficiais desses materiais, incluindo a sua mobilidade (dinâmica molecular). Na escala microscópica sabe-se, por exemplo, que a tensão superficial dos materiais possui uma dependência espacial (dependência do vetor de onda $q$ ) devido ao efeito de interação superficial entre a matéria mole e o substrato (por exemplo, um filme de poliestireno que adere a um capilar). A técnica de XPCS pode acessar tempos de até $\mathrm{t} \sim 10^{\circ} \mathrm{s}$, característicos, por exemplo, da mobilidade de poliestireno em meio capilar, cujo comportamento dinâmico pode ser descrito pela mecânica dos fluídos, facilitando a interpretação teórica dos resultados.

Por outro lado, a aplicação de campos externos (elétricos, magnéticos etc.) a sistemas poliméricos pode ser um método eficiente para controlar a organização dos microdomínios nessas estrutu- 
ras compostas de matéria mole. Dessa forma podem-se induzir morfologias e diferentes dimensōes em arranjos moleculares característicos de separação de microfases. Resultados recentes (13) indicam que é possível o estudo da indução de transições de fase em suspensões coloidais, aplicando-se um campo elétrico externo acoplado às medidas com a técnica de SAXS. Nesse estudo (13), o copolímero dibloco poli (estireno-b-etileno-co-propileno) (PS-b-PEP) foi dissolvido em uma mistura de solventes (cicloexano e dimetilformamida). Uma vez que os solventes puros são imiscíveis e os diferentes blocos do copolímero interagem preferencialmente cada um com um dos solventes, na temperatura ambiente origina-se uma estrutura cúbica em 3 dimensões (3D). Nessa estrutura em 3D, os microdomínios esféricos de dimetilformamida ficam envolvidos em uma matriz líquida de cicloexano, sendo que a interface líquido-líquido encontra-se recoberta pelas longas cadeias do copolímero em bloco PS-b-PEP, em função das características de hidrofobicidade dos blocos. Uma vez aplicado o campo elétrico no experimento de SAXS, os resultados (combinados com a técnica similar que usa feixes de nêutrons, SANS) demonstraram que a estrutura cúbica havia se convertido em um arranjo hexagonal. Sob um campo externo, os microdomínios esféricos de dimetilformamida são deformados e interconectados levando à formação de cilindros, com organização hexagonal. Devido ao grande contraste dielétrico entre a matriz líquida de cicloexano $(\varepsilon=2.0)$ e os microdomínios contendo a dimetilformamida $(\varepsilon=38.0)$, um campo elétrico da ordem de $\sim 1.25 \mathrm{kV} / \mathrm{mm}$ foi suficiente para que ocorresse uma transição de fase do arranjo cúbico para hexagonal, no seio da suspensão coloidal. A técnica de XPCS poderá ser útil na elucidação dos tempos característicos para a completa transformação das estruturas (reorganização dos microdomínios 3D para arranjo hexagonal), em função do campo elétrico aplicado e da temperatura.

Sabe-se que massas fundidas de copolímeros em bloco podem formar microestruturas tais como esferas, cilindros e lamelas, bem como estruturas bicontínuas mais complexas, dependendo da razão de comprimento dos blocos, da interação intermolecular entre os copolímeros ou da presença de homopolímeros adicionais (14). Nesses casos há necessidade de investigar a resposta dinâmica e reológica desses fluidos complexos, o que requer informações experimentais sobre as escalas microscópicas de comprimento que caracterizam suas estruturas hierárquicas. A técnica de XPCS é capaz de revelar a dinâmica desses sistemas, que se caracterizam por escalas de comprimento relativamente pequenas e escalas de dinâmica temporal relativamente longas, tratando-se nesses casos de dinâmica estrutural. Informações relevantes sobre o comportamento viscoelástico desses sistemas podem ser obtidas (14).

A técnica de XPCS também permite o estudo de sistemas vítreos e géis. Do ponto de vista da reologia desses sistemas, a técnica permite o estudo da dinâmica desses materiais fluidos e desordenados, principalmente em suas transições de fase características. Microsco- picamente, transições vítreas e gelificação originam fenômenos de espalhamento múltiplo, o que é um grande problema experimental na investigação da dinâmica desses sistemas por DLS. Por outro lado, o fenômeno do espalhamento múltiplo não afeta a técnica de XPCS. A compreensão da relação entre a dinâmica desses sistemas (vidros e géis) e suas transições de fase são os principais desafios a serem elucidados, o que pode ser estudado através de XPCS (14).

Sistemas coloidais mais complexos A técnica de SAXS não requer, a priori, que as amostras sejam transparentes, como nas medidas com as técnicas de espalhamento de luz. Dessa forma, pode ser empregada para o estudo de sistemas coloidais mais complexos como amostras de petróleo, por exemplo. Neste caso, medidas com SAXS (e com a técnica similar que usa feixes de nêutrons, SANS) mostram que amostras de petróleo contém partículas coloidais, em alguns casos formadas por agregados de moléculas polares chamadas de asfaltenos (15). Na tentativa de compreender a origem microscópica da alta viscosidade dos óleos brutos pesados, medidas macroscópicas (mediçôes reológicas) e microscópicas (SAXS) são aplicadas. Estudos recentes (15) indicam uma clara relação entre o conteúdo de asfalteno e a viscosidade do óleo. Por outro lado, a remoção de asfalteno por floculação leva a uma grande queda de viscosidade, confirmando o asfalteno como a origem da alta viscosidade nesse meio. As análises SAXS dos óleos brutos confirmaram a presença de agregados de asfaltenos como partículas de dimensōes coloidais de tipo fractal. $\mathrm{O}$ estudo desses agregados é importante porque sua deposição pode levar a problemas de entupimento na rocha de onde o petróleo é extraído ou em tubulações durante sua extração, sendo por isso comparado com o colesterol para a saúde humana.

Nanotubos de carbono são um outro exemplo de materiais auto-organizados utilizados na preparação de sistemas de interesse tecnológico. Estes têm atraído muita atenção devido à sua aplicabilidade na eletrônica, na mecânica e na ótica. A partir da década de 1990, especialmente os nanotubos de carbono de parede única (SWCNT) têm sido bastante investigados. Um estudo envolvendo peptídeos fotoativos ancorados sobre nanotubos de carbono (SWCNT) demonstrou que, em solução, esses nanotubos se associam, mediados pelas macromoléculas de peptídeo (16). A técnica de SAXS permitiu a determinação da espessura dos SWCVT $(8,97 \mathrm{~nm})$, bem como a determinação da existência de estruturas agregadas (2D) em solução, compostas de nanotubos de parede única (SWCNT). Com base nos resultados, os autores sugerem que a excelente estabilidade encontrada nos sistemas é aumentada pela ordem de longo alcance detectada por SAXS. Indicam ainda que esses compósitos com organização na mesoescala podem ser transferidos para substratos sólidos, para serem utilizados como modelos para o controle de morfologias em uma variedade de dispositivos eletrônicos, cuja eficiência é altamente influenciada pela organização molecular intrínseca (16).

Amostras de solo constituem outro sistema coloidal em que SAXS é uma ferramenta fundamental, uma vez que o tamanho das estru- 
turas porosas e a área superficial das partículas ali presentes são fundamentais para o sequestro e o transporte de carbono (17). O solo contém coloides que podem ser facilmente dispersos em contato com a água presente no material. São partículas com dimensões inferiores a $2 \mu \mathrm{m}$ e incluem principalmente aluminosilicato e partículas de óxidos metálicos que podem ser liberados a partir de estruturas agregadas do solo, promovendo a dispersão da matéria orgânica. Medidas de SAXS nesses sistemas permitem comparar o efeito de colóides metálicos na capacidade de compactação dos solos, bem como a rugosidade característica, em função da natureza fractal dos materiais dispersos. Esse estudo propõe que o empacotamento das nanopartículas e a sua área superficial são determinantes na capacidade de retenção de carbono desses solos, indicando a importância dos resultados fornecidos pelas análises de SAXS. Também pelo benefício da penetração dos raios $\mathrm{X}$ em amostras escuras e concentradas, a técnica de XPCS poderá ser utilizada em sistemas coloidais complexos desse tipo (solos) para avaliar a dinâmica desses agregados e partículas fractais, responsáveis pelas propriedades reológicas desses sistemas.

PERSPECTIVAS Na breve descrição acima procuramos demonstrar a importância das técnicas de espalhamento de raios X em ângulos baixos (SAXS) e espectroscopia de correlação de fótons de raios $\mathrm{X}$ (XPCS) no estudo de sistemas importantes em nanociência. Os materiais apresentados podem ser classificados como matéria mole e são compostos por moléculas orgânicas a partir da sua organização supramolecular que ocorre por interações não covalentes. Esses sistemas envolvem surfactantes, polímeros (em alguns casos biopolímeros) e outras moléculas, produzindo sistemas complexos que normalmente apresentam um arranjo estrutural característico. Pelas suas dimensões coloidais - na faixa de nanômetros (nm) a micrômetros $(\mu \mathrm{m})$-, essas estruturas se situam na faixa de tamanho ideal para a aplicação da técnica de SAXS. Investigações utilizando SAXS, muitas vezes aliadas ao ajuste das curvas obtidas utilizando modelos teóricos, fornecem não apenas o tamanho e a forma dos objetos nanométricos, como também informações sobre o seu arranjo espacial. Dessa forma, essas técnicas permitem entender os processos envolvidos na associação molecular e supramolecular e, a partir dessas informaçōes, imaginar aplicações que se beneficiem de suas estruturas e propriedades.

É interessante ressaltar que, em alguns sistemas coloidais, esse arranjo de nanopartículas pode ser associado ao arranjo de átomos para formar moléculas, as chamadas "moléculas coloidais" (18), o que levou Tomalia e Khanna a propor a busca por arranjos nano-periódicos como um conceito unificador em nanociência (19). A maioria das rotas sintéticas para a preparação desses novos materiais consiste, por um lado, no controle do processo de formação de agregados por meio de coalescência, rotas físicas e químicas e confinamento em múltiplas dimensões e, por outro lado, no uso de fenômenos bem conhecidos como separação de fases ou mecanismos de nucleação e crescimento controlado de agregados. O estudo da dinâmica de processos de formação de "moléculas coloidais" uti- lizando experimentos de SAXS e XPCS poderá levar ao descobrimentos de processos e configurações intermediárias que não podem ser observadas por outro método.

A divulgação das técnicas de espalhamento de raios $\mathrm{X}$ em ângulos baixos (SAXS) e espectroscopia de correlação de fótons de raios $\mathrm{X}$ (XPCS), bem como das informaçōes que permitem obter, é muito importante para o desenvolvimento da nanociência no Brasil, principalmente se considerada a infraestrutura já em funcionamento em laboratórios como o LNLS ou, no caso de XPCS, sua futura disponibilidade dentro das linhas a serem instaladas no laboratório Sirius.

Watson Loh é químico, professor titular do Instituto de Química da Universidade Estadual de Campinas (Unicamp). Foi diretor do Instituto de Quimica da Unicamp. Atualmente é editor-chefe da revista Journal of Brasilian Chemical Society (JBCS)

Nádya Pesce da Silveira é química, professora titular do Departamento de Química Inorgânica do Instituto de Química da Universidade Federal do Rio Grande do Sul (UFRGS). Foi diretora científica e diretora presidente da Fundação de Amparo à Pesquisa do Estado do Rio Grande do Sul (Fapergs). Atualmente é diretora do Instituto de Química da UFRGS.

\section{REFERÊNCIAS}

1. Engelskirchen, S.; Kulkarni, C. V. Phys. Chem. Chem. Phys., 13, 3003, 2011.

2. De Gennes, P. G. Science, 256, 495, 1992.

3. Narayanan, T.; Current Opinion in Colloid \& Interface Science, v. 14, n. 6, 409, 2009.

4. 〈http://Inls.cnpem.br/sirius/〉. Acesso em março de 2017.

5. Borsali, R.; Pecora, R. (editors); Soft-Matter Characterization, Springer, 2008.

6. Craievich, A. F. Materials Research, v. 5, n. 1, p. 1, 2002.

7. Sutton, M.; C.R. Physique, 9, 657, 2008.

8. Jensen, G. V.; Lund, R.; Gummel, J.; Narayan, T.; Pedersen, J. S. Angew Chem., v. 53, n. 43, p. 11524, 2014.

9. Ferreira, G. A.; Piculell, L.; Loh, W. ACS Omega, v. 1, n. 6, p. 1104, 2016.

10. Mertins, O.; Cardoso, M. B.; Pohlmann A. R.; Silveira, N. P. J. Nan. Nanotech., 6, 24, 2006.

11. 〈http://revistapesquisa.fapesp.br/2009/05/01/carapaca-versatil/〉. Acesso em março de 2017.

12. Hoshino, T.; Tanaka, Y.; Jinnai, H.; Takahara, A.J Physical Soc. Japan, v. 82, n. 2, p. 021014, 2013.

13. Giacomelli, F. C.; Nallet, F.; Černoch, P.; Steinhart, M.; Štěpánek, P.; Silveira, N. P. Macromolecules, 43, 4261, 2010.

14. Leheny, R., L. Current Opinion in Colloid \& Interface Science, v. 17, n. 1, p. 3, 2012.

15. Padula' L.; da Silveira Balestrin, L. B.; Rocha, N. O.; Monteiro de CarvaIho, C. H.; Westfahl Jr., H.; Cardoso, M. B.; Sabadini, E.; Loh, W. Energy Fuels, v. 30, n. 5, p. 3644, 2016.

16. Brunetti, F. G.; Romero-Nieto, C.; Lopez-Andarias, J.; Atienza, C.; López, J. L.; Guldi, D. M.; Martin, N.; Angew Chem., v. 52, n. 8, p. 2180, 2013.

17. Jiang, C.; Séquaris, J. M.; Wacha, A.; Bóta, A.; Vereecken, H.; Klumpp, E. Geoderma, 260, p. 235-236, 2014.

18. Duguet, E.; Desert, A.; Perro, A.; Ravaine, S. Chem. Soc. Rev. 40, 941, 2011.

19. Tomalia, D. A.; Khanna, S. N. Chem. Rev., v. 116, n. 4, p. 2705, 2016. 